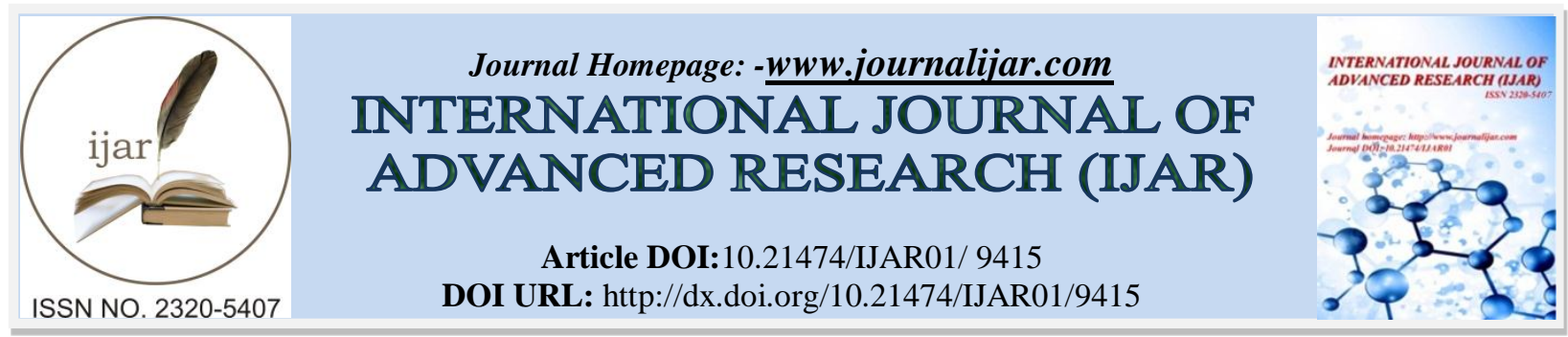

RESEARCH ARTICLE

\title{
DIGITAL SIGNATURE METHODS BASED BIOMETRICS.
}

Shaimaa h. Shaker And ArwaS.Ridha.

Computer Sciences Department, University of Technology.Baghdad / Iraq.

\section{Manuscript Info}

Manuscript History

Received: 20 May 2019

Final Accepted: 22 June 2019

Published: July 2019

Key words:-

Digital signature, eye movement, pupil movement, biometrics measures, authentication.

\begin{abstract}
Digital signature is an encrypted communication method that documents and authenticates online transactions around the world and is used to authenticate user identity. The purpose of this paper is to inventory most of the existing techniques that previous researchers have found on the subject of signature verification based on most of the various human biometric features and compare these studies to achieve the best performance and best method of digital signature work in conjunction with biometric features. Through a review of literature and previous research and analysis and comparison, it was concluded that the digital signature based on eye movement tracking is very useful and efficient and fast and serves people with disabilities who cannot interact with the computer naturally and help them to authenticate their identities.
\end{abstract}

Copy Right, IJAR, 2019,. All rights reserved.

\section{Introduction:-}

Biometric authentication system is a system that provides the user process to identify and verify the user's identity and authenticate using dynamic features of the user because it has unique characteristics distinguish each user from another. These features are shared in many different fields such as e-commerce, forensic science, engineering, border control, etc. These biometric features must be perfect and unique, they can be evaluated easily, they are costefficient and have great user approval. In fact, not all biometrics meet all of these requirements at the same time. Therefore, to obtain the highest attainment of the requirements, More than one biometric feature is integrated into a single security system for a higher level of security, reliability and authentication. Integration may not meet all requirements of biometric systems. Given the technological development of the world and the race of science. Especially when we are talking about information security systems related to state institutions, economy, weapons etc. We see the importance of using and using new methods that may be less vulnerable to infringement and more security. One of these methods is digital signature that provides security requirements, achieves high reliability and authentic authentication of the person and ensures The purpose of this research is to identify most of the current techniques found by previous researchers in the subject of signature verification based on most biometric properties And compare these studies to achieve the best performance and the best way. For digital signature work combined with biometric features. The biometric authentication process for the digital signature is divided into two tasks: first, user identification. In this process, an unknown biometric sample is matched with a database containing pre-defined biometric samples; secondly, verification of the user's identity is verified in the process if the claimant is the same person claiming that in both cases no decision is made[1]. 
Biometric features are divided into several categories such as fingerprint recognition, voice recognition, iris, pupil, ear, tongue, DNA and other biometric systems. However, many individual biometric systems have been violated, such as fingerprint and iris, so we consider it necessary to use Another method is safer and less vulnerable to violation than previous methods As a result, digital signature work relies on eye tracking technology, making images or coatings through eye movement more effective than previous methods and gives better results in terms of safety, reliability and authentication [2].

\section{Biometric Digital Signatures [4]:}

A specific type of sensor or device system are needed by the majority of biological data identifiers for securing and controlling the identity of individuals. Each individual has inadequate biological measurements, in the case when the biological data is inadvertently accessed or exposed, the identity related to the individual with the exposed biological measurements cannot be utilized for the purpose of validation once more. For addressing such issue, some cancellation approaches were presented. Among the various biometric approaches, signature is considered to be only reversible from the deception perspective, in the case when the signature form is recognized and exposed, the issue could be handled via changing the form, in such systems which challenge aspect in autorun signature authentication is necessity for obtaining obtain results of high-resolution for the purpose of avoiding refusal or wrong authorization. While the signature verification system decide if the specified signature belong to the individual claiming such signature-based authentication cannot be static (digital signature only available) or dynamic (signatures are obtained via a drawing board or sensitive to the pen the computer display is A biometric feature has been established as program of engine control, executed via nervous system, left on writing surface via hand-writing device, thus the verification as well as signature recognition is of high importance. The devices of signature verification are precise in process and clearly provide applications for applications where the signature is acceptable and the handwritten signature is the most efficient biometric approach to identify persons, also the most widely accepted and sometimes the simple written signature is used as a way to assert the identification in addition to permitting access to the system. However, signatures are considered to be variable and complex to distinguish, subject to fraud as well as to abuse, and technological issues represented via signature verification Automatic task, the following fig (5) shows the flowchart of a Signature Verification System.

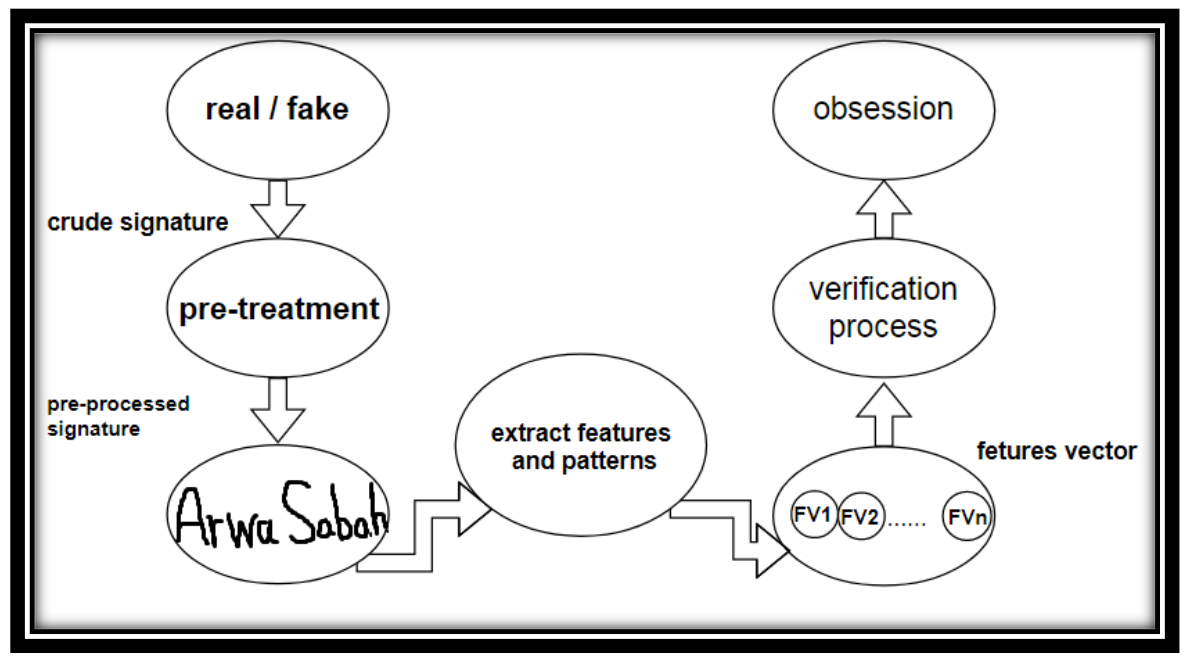

Figure 1:-Flowchart of a Signature Verification System.

\section{Biometric Measures:}

Biometric systems can be defined as a technology that is progressing, also they were utilized in various commercial and official identification applications. Biometric technique is mainly a pattern recognition system that make personal identification decision via deciding the authority regarding particular behavioral or physiological traits.

There is drawbacks and advantages in all biometric technologies, choosing a biometric measure is done based on particular application. It unexpected that single biometric trait is going to effectively achieve all the requirements of applications (cost, practicality and precision). Some biometric features were used for various applications for the purpose of obtaining more efficient results, Fig (1) show a mechanism or scheme that is applicable on the biometric of individuals [4]. 


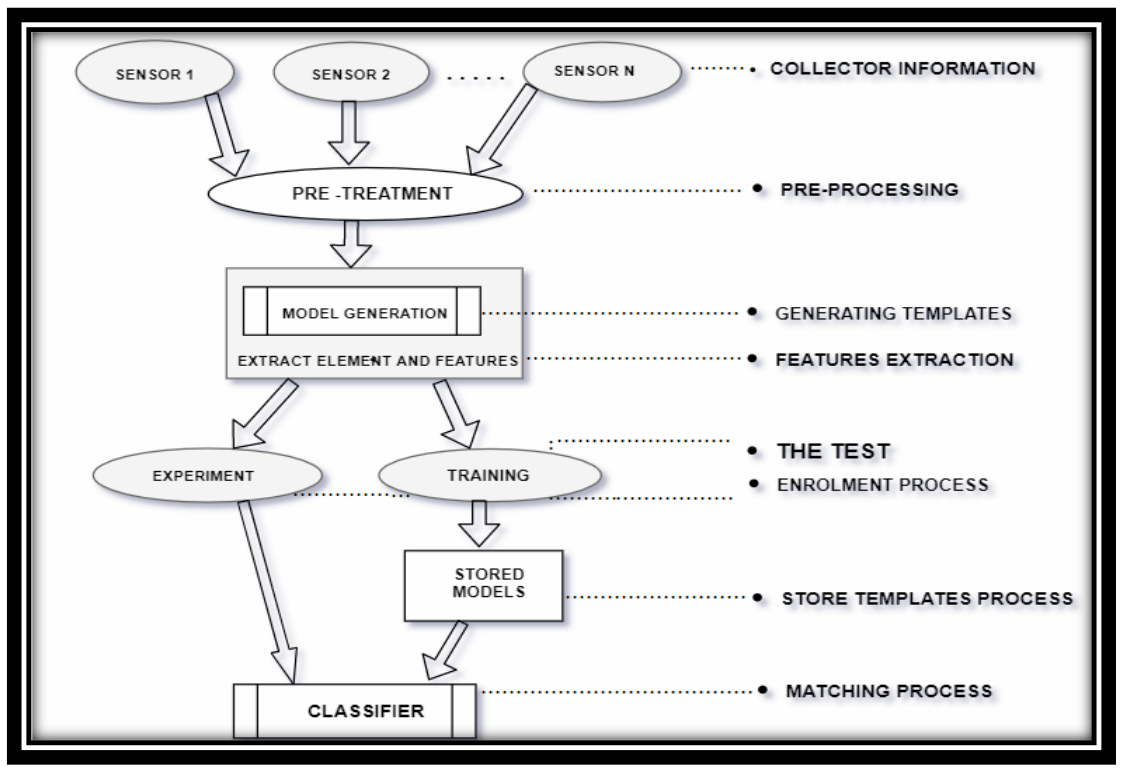

Figure 2:-Framework of human biometric system.

A lot of biometric measurements were discovered, and now we will briefly discuss some of the most important biometric features:

Hand geometry [4]:

This feature is considered as biological measure which utilizes the geometrical shape that is related to the hand with the shape of the hand, the width of the fingers, the palm's size and lengths [1] to verify the identity of the user. These biometrics provide optimum balance in the performance characteristics, also they could be used easily. In contrast, hand engineering is affected by several things such as dry wind, dry skin, deformities and changes in the hand. Furthermore, the information related to hand geometry could not be constant throughout the growth of children until youth, the ornaments of the individual could indicate additional issues when extracting the precise information of hand geometry.

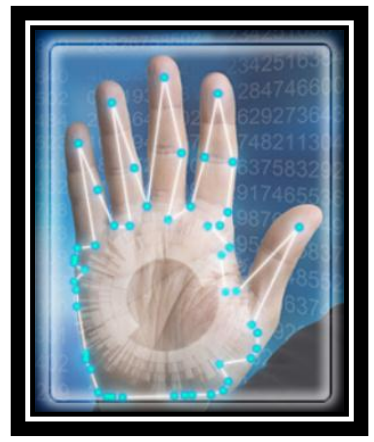

Iris of the eye[4]:

Iris can be defined as the eye's area, it is surrounded via pupil and solid (white eye) on the two sides, the development regarding the iris patterns following 6 months of birth, they become stable after 1 year. Then, patterns will not change anymore. The issue of iris have extremely important information and it is of high importance in personal recognition; the iris strength is extremely difficult to manipulate surgically. Even though that the previous iris-based identification systems were expensive and need large participation of users, the novel approaches are more cost-efficient and affordable.

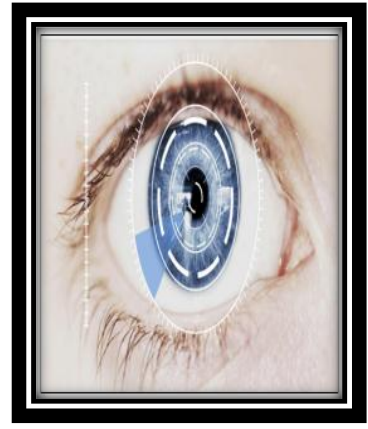




\section{Fingerprint[4]:-}

One of the major biometrics used for identifying the users and verifying their identity is fingerprints, the characteristics of fingerprints remain fixed and unique throughout the life of any person has been used by the human identification for several decades, but on the other hand, they are affected by any abnormalities or injuries can affect the fingers such as burns, Wounds and amputations as well as any insulating material may affect fingerprints.

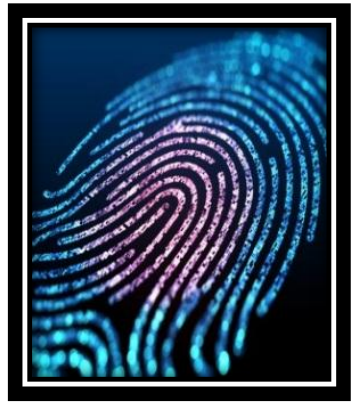

Face[4]:

One of the main biometric features related to identifying individuals is facial images. The identity of the person is automatically identified from a digital photo, providing constant authentication of the person's identity by identifying facial properties (chin, eyebrows, lips, eyelid, eyes, and nose). Of the advantages associated with it is directly linked to security, but any change the features of the face or hide all or part of the face affects the process of identifying the person.

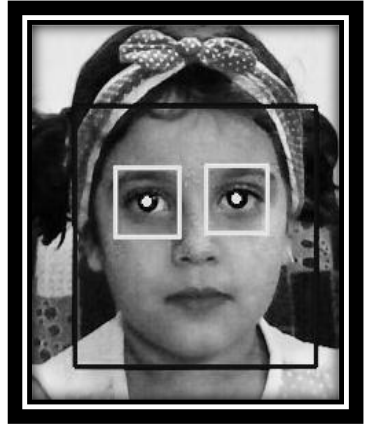

\section{Voice[4]:}

This features is considered as a combination of behavioral and physical biometrics. Sound is converted to text where the voice features of the individual depend on size and shape (lips, mouth, voice, and nose) which are utilized for synthesizing the sound. Such properties are fixed for the individual, but the behavioral part of the speech of the individual will be changing with time because of age, emotional state, health conditions (colds), and sound are also not very distinctive and may not be suitable for broad identification.

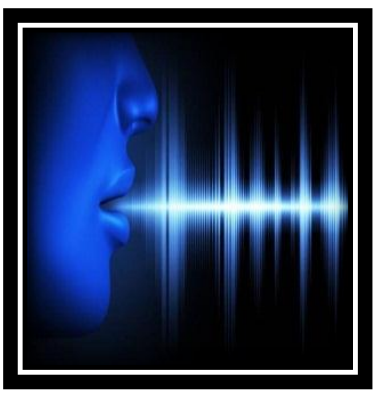

\section{Retina[4]:}

Retinal-based biometrics incorporate the vascular coating found in the back of the eye. This technique involves the use of a light source of high-intensity via light coupler for scanning distinctive retinal patterns. It can be completely accurate examination of the retina, but it requires the user to look and concentrate on particular point. This cannot be 
achieved if glasses are used or concerned on close connection to reading device. Also, Retina scanning is unacceptable by all users.

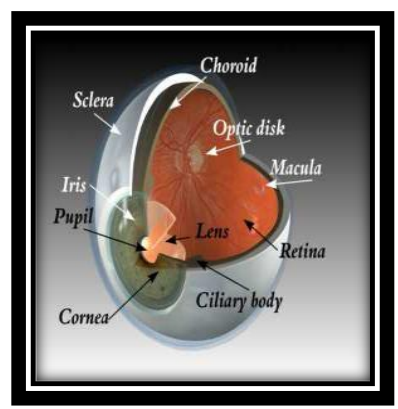

Keyboard (behavioral biometric)[4]: There is an assumption that there is a distinctive approach each individual use when typing on the keyboard. Such behavioral biometrics are unexpected to be distinctive to each person but are unexpected to offer adequate discriminatory information for permitting verifying the identity. The dynamics of compression keys are dynamic behavior scale and there are enormous differences in the typical writing patterns.

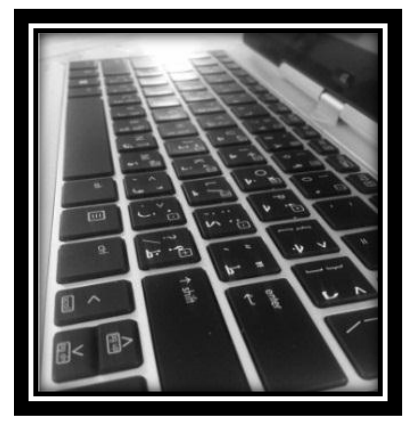

\section{Sclera[4]:}

Identifying a sclera one important features are protected very eye part, it is very difficult to spoof. Can identify someone by patterns in sclera container because it has these patterns with a high degree of randomness and this makes it ideal for personal identity also remains constant patterns throughout the life of the person. You can integrate this feature easily with dynamic measurements of the iris.

\section{i- Saccades[3]:}

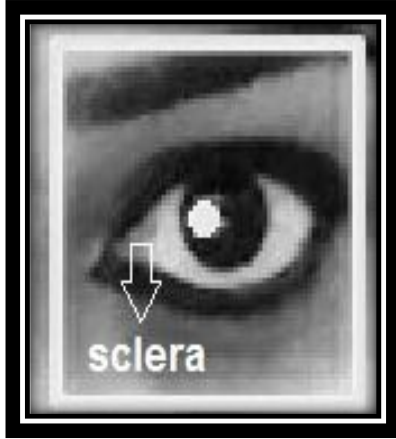

Saccades are important as a biometric data exporter, it is the fastest eye movements carried out by people, almost durations are approximately $(0.1$ to 0.4$)$ in the second, there is a high capacity saccade and low amplitude saccade, is used in general medicine as well as useful and very important in operations such as identity verification and identification of the user.

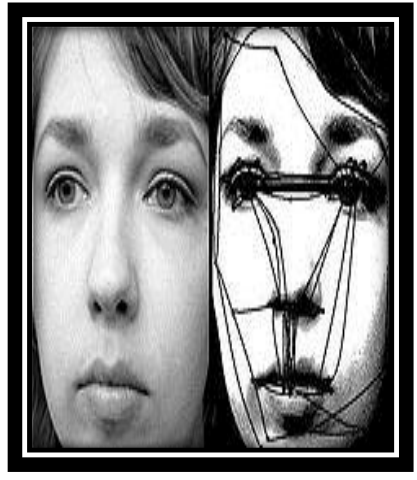




\section{Eye movement tracking system:}

In order to determine the eye movement of a specific object, you must first determine the object's head and then identify the face of the object and finally enter the details of the face and identify the object's eye, Individuals move their eyes for the purpose of bringing an image of an object onto fovea.[1].

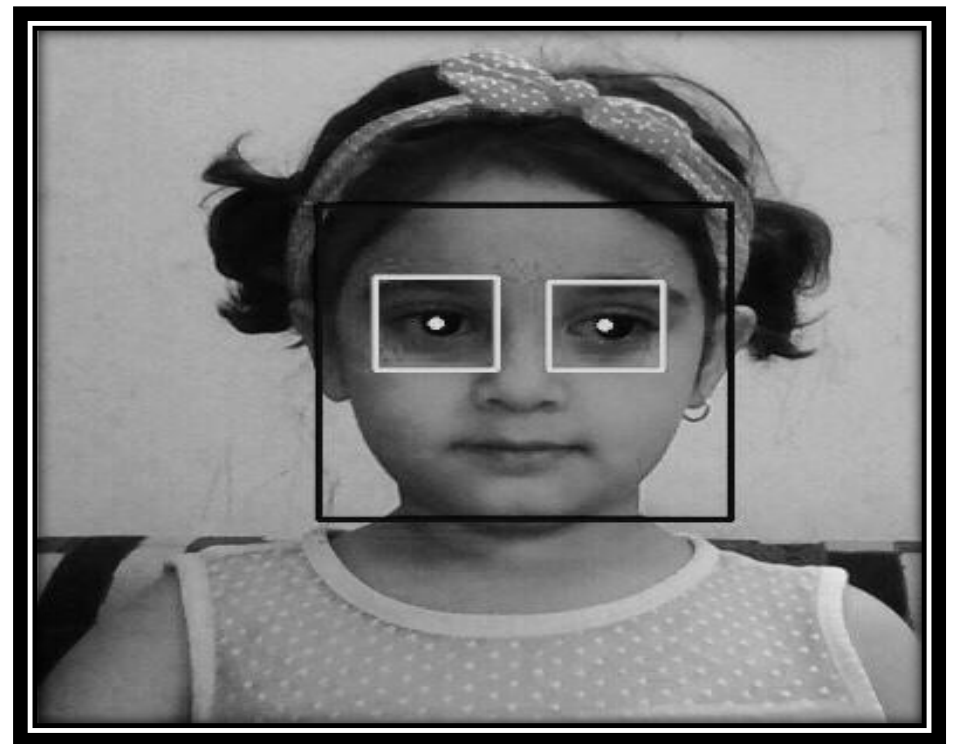

Figure 3:-human face detection and eye movement.

As soon as the object's image is stabilized on retina, one can extract the information. Such movements could be recorded via the device (Eye-tracker). For the purpose of estimating the gaze's direction, eye-tracker utilize infrared light emitter and video image analysis regarding the center of the pupil and reflections from cornea. [1]

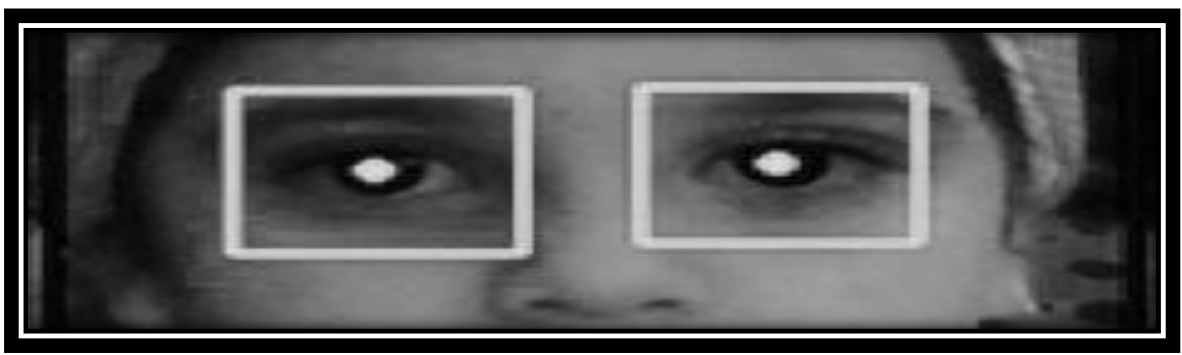

Figure 4:-Eye movement tracking of human eyes.

Eye-tracker can be defined as a device that record the movements of the eye. For the purpose of estimating the direction of view, the eye tracker utilize infrared light emitter; analyzing the video images regarding the center of the pupil, as well as the eye's reflections. Cornea. Previous research has shown strong evidence of the relationship between basic cognitive process, visual attention and eye movement. As in the eye, the diameter of the pupil is not constant but fluctuates around a specific value. These systems are unique and unique. of biometrics. These systems use video imaging for detecting the eye's position. As a result, there is the possibility of incorporating such biometrics into a single eye-based system. These systems can also be integrated with recognition systems, although eye tracking devices are expensive, that the cost of technology is lower. This gives the possibility of using eye tracking standards with other biometrics [1].

In order to trace the human eye, it must pass through several stages such as:

1. Object detection

2. Head detection

3. Face detection

4. Eye detection [10]. 


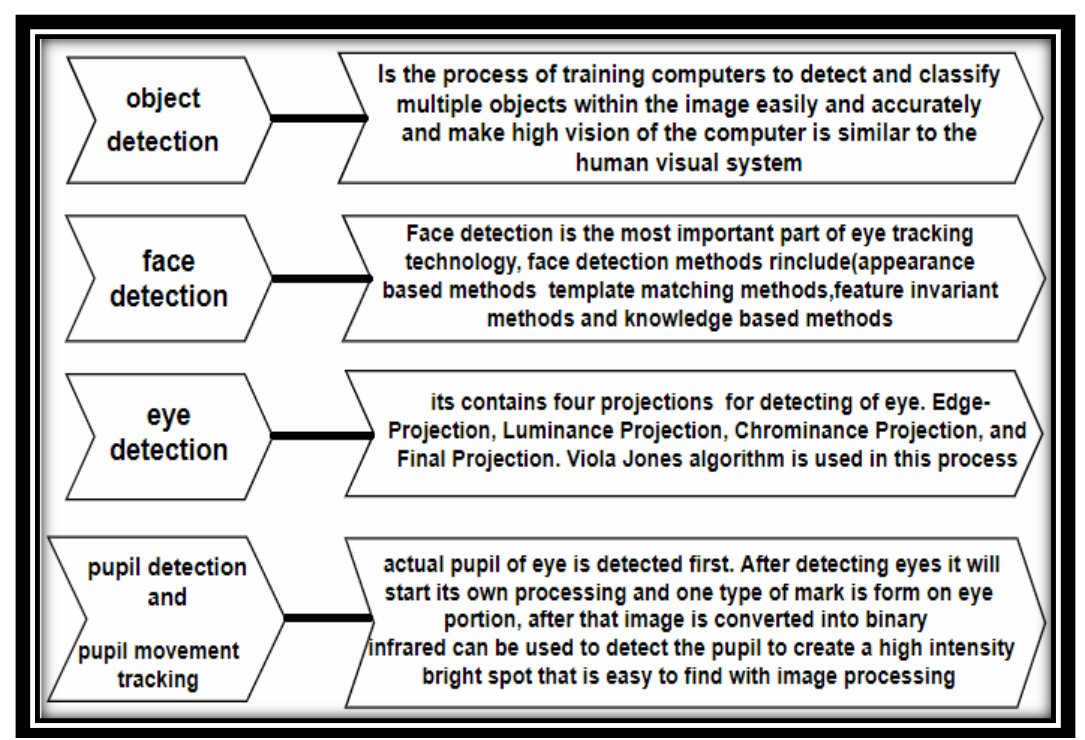

Figure 5:-Eye movement tracking system.

\section{Related work:}

This paper examines security systems and digital signature systems that rely on most of the different biometric features developed by different researchers according to their experience. The following paragraph shows the most important previous published studies related to the purpose of this research.

1. The Romans, Canon, André, and pasi introduced ways to use eye movements as dynamic measurements. More than one feature is integrated. The potential related to the eye data movement for biometric intentions has been examined. The optimum dynamic feature has been the size of Delta pupil (60\%). Time is interesting and interesting, that the size of the student himself provides a somewhat weaker discrimination (40\%). Combining various peripheral features provided only an enhancement in the precision of identification, the results have indicated that there is discrimination in the movement of the eye [1].

2. Carlos, Francisco, and Rogelio (2017) present "scan-path comparison" A method is used to compare the scan path to authenticate people by their eye movement, then their eye movements are recorded using an eye tracking device and a PIN is drawn on the screen. theeyenalysis algorithm was used to measure the similarity of the scan paths by calculating the distance in pixels per point on the path.[2].

3. Daniel, M. Angela and john presented a Study on the use of eye tracking as a data collection tool, also an overview related to the data of eye movement that could be collected, what it can add to the present HCI study and what is involved in analyzing. Furthermore, this approach have the ability of providing useful objective data on the impact of visual design on human performance.[6].

4. Srikanta ,Umapada and Michael (2014) present “ signature-based biometric authentication”, These researchers provided detailed and accurate research on signature-based biometric authentication. This study included several areas such as the automatic signature verification system, the concept of biometric system, the concept of signature verification and automatic verification of signature, which is a very interesting field in scientific terms. For continuous signature and renewables along with improved security Among all the vital features, longer signature verification work is very important in the field of biometric measurements.[4].

5. Justin, Kenrick and Bogdan (2011) present method of "EyeDent System" This system is an improvement to the current authentication systems that depend on eye tracking technology that requires pressure on the operator by the person in case of looking at any code on the screen. Instead, EyeDent method automatically assembles the gaze points to select the object chosen by the person; this technique is useful because there is no visible trigger where it does not detect how many symbols exists in the password and it allows the person to authenticate as quickly as he chooses, instead of the specified time In advance.[5].

6. Andreas and Rejean(2016) presentedA system to verify the signature based on the interaction between the user and his movements using smart devices such as the use of pen or thumb movement by analyzing these movements using the kinematic theory of rapid human movement by direct comparison of the elementary neuromuscular strokes which are detected in the handwriting.[7].

7. Yousef,Keivan, A Motie and Mohammad presented a method for estimating elasticity and viscoelasticity characteristics of the muscles and tendons of hand human, In addition, the NMC model was used to describe the 
dynamics of the human joint (the human finger), the parts has been description by a frequency field which used by the frequency response function (FRF), the index finger of the right hand was presented and the angles between the finger knuckles were collected by data collection glove and the location of digital pen tip on sensitive pad is stored in computerThis approach has been applied on 30 right-hand persons. This method has been used to complement the techniques of signature verification by focusing on the person's physiological muscles during the signature.[8].

8. Alexander, Martin and Heinrich (2009) present "Eye-PassShapestechnique", Eye-PassShapes extends and develops two authentication approaches via combining them, EyePIN andPassShapes. EyePIN is focused on security instead of usability.The user's PIN is still the token of authentication, and the security is improved when the input method is changed. Rather than inserting numbers, an eye movement is performed by the the user representing the associated digits. In PassShape the users must paint shapes (that consist of strokes) in a certain order, this method increase memorability but doesn't improve security in comparison with PIN or password entry, Eye-PassShapes can be considered simpler to be detected than the exact location of the user's look and can work with cheap devices. [9].

9. Kunal ,kiran and Mrs.sunitajadhav present “ eye task"system, A human computer interaction system designed for people with severe disabilities and those with different spinal injuries who can only control their eyes. This system makes them able to communicate with the computer and make decisions using their eyes.[10].

\section{Comparison between Biometric Approachs:}

This section provides some details about some studies and previous work related to security systems and digital signature systems based on different biometric features as shown in Table [1].

Table 1:-List of most references and studies provided to strengthen security systems and digital signature systems based on biometric features and the processes of improvement and development achieved.

\begin{tabular}{|c|c|c|c|c|c|c|}
\hline $\begin{array}{l}\text { Researcher } \\
\text { name }\end{array}$ & year & $\begin{array}{l}\text { Method } \\
\text { (technique) }\end{array}$ & \multicolumn{4}{|l|}{ Pros and Cons } \\
\hline $\begin{array}{l}\text { Daniel,M. Angela and } \\
\text { John.[6]. }\end{array}$ & 2002 & $\begin{array}{l}\text { Eye tracking as } \\
\text { a data collection } \\
\text { tool. }\end{array}$ & \multicolumn{4}{|c|}{$\begin{array}{l}\text {-The close relation between the data of eye tracking and } \\
\text { the subjective reports provides certain substance to our } \\
\text { argument that there should be mutual dependence on } \\
\text { objective and subjective measures in HCI evaluation } \\
\text { approaches. } \\
\text {-The results showed a relationship between GSR and eye } \\
\text { movements. }\end{array}$} \\
\hline \multirow[t]{4}{*}{$\begin{array}{l}\text { Roman, kinnunen, Andrei } \\
\text { and pasi [1]. }\end{array}$} & \multirow[t]{4}{*}{2005} & \multirow{4}{*}{$\begin{array}{l}\text { Eye movement } \\
\text { as biometric } \\
\text { features }\end{array}$} & Method features & FFT & PCA & \begin{tabular}{l|l} 
FFT \\
$+\mathrm{PCA}$
\end{tabular} \\
\hline & & & Pupil +velocity & 42 & 42 & 42 \\
\hline & & & Pupil +distance & 90 & 90 & 90 \\
\hline & & & $\begin{array}{l}\text { Pupil+ } \\
\text { velocity+distance }\end{array}$ & 92 & 88 & 92 \\
\hline $\begin{array}{lll}\text { Alexander, } & \text { Martin } & \text { and } \\
\text { Heinrich [9] } & & \end{array}$ & 2009 & $\begin{array}{l}\text { Eye-PassShapes } \\
\text { technique }\end{array}$ & \multicolumn{4}{|c|}{$\begin{array}{l}\text {-EyePassShapes have higher security than the PassShapes. } \\
\text {-EyePassShapes can be considered slower than the } \\
\text { standard PIN-entry. } \\
\text {-EyePassShapes is considered to be simpler to utilize than } \\
\text { EyePIN. } \\
\text {-EyePassShapes is considered to be faster than EyePIN. } \\
\text {-PassShapes utilizing EyePassShapes are as memorable as } \\
\text { PassShapes utilizing touchpad. } \\
\text {-EyePassShapes have higher security the than standard } \\
\text { PIN-entry. } \\
\text {-PassShapes utilizing EyePassShapes with the repeated } \\
\text { input strategy is considered to be simpler to remember } \\
\text { than without. }\end{array}$} \\
\hline $\begin{array}{l}\text { Justein,kenrick and Bogdan } \\
\text { [5] }\end{array}$ & 2011 & Eyedent system. & \multicolumn{4}{|c|}{$\begin{array}{l}\text {-Enhance the authentication process } \\
\text { by allowing individual errors such as an alternate } \\
\text { character or a inserted or missing character. }\end{array}$} \\
\hline
\end{tabular}




\begin{tabular}{|c|c|c|c|}
\hline $\begin{array}{l}\text { Srikanta,Umapada and } \\
\text { Michael[4] }\end{array}$ & 2014 & $\begin{array}{l}\text { automatic } \\
\text { signature } \\
\text { verification } \\
\text { system }\end{array}$ & $\begin{array}{l}\text { - Authentication can be achieved } \\
\text { without a sensor } \\
\text {-Make a high effort to resolve the problem of } \\
\text { authentication. } \\
\text { - The signature authentication system could have high } \\
\text { level of accuracy similar to other biometric systems. }\end{array}$ \\
\hline Andreas and Rejean.[7]. & 2016 & $\begin{array}{l}\text { kinematic } \\
\text { theory of rapid } \\
\text { human } \\
\text { movements }\end{array}$ & $\begin{array}{l}\text {-The model quality and performance verification are } \\
\text { promising for the improvement of data sets. } \\
\text {-improve the overall quality of the extracted strokes from } \\
\text { a neuromuscular perspective not only the SNR. }\end{array}$ \\
\hline $\begin{array}{l}\text { Carlos, Francisco, and } \\
\text { Rogelio.[2]. }\end{array}$ & 2017 & $\begin{array}{l}\text { Eyenalysis } \\
\text { algorithm }\end{array}$ & $\begin{array}{l}\text {-Get the best results for people who } \\
\text { have already been trained. } \\
\text {-Obtain an average acceptance rate of } \\
80 \% \\
\text {-The low error rate of } 25 \%\end{array}$ \\
\hline $\begin{array}{l}\text { Yousef, Keivan, A Motie } \\
\text { and Mohammad.[8]. }\end{array}$ & 2017 & $\begin{array}{l}\text { Physiology of } \\
\text { hand and tendon } \\
\text { muscles ,NMC } \\
\text { model }\end{array}$ & $\begin{array}{l}\text { - from } 30 \text { right-hand persons, that of each individual } 5 \text { real } \\
\text { signature and some ordinary fake to counterfeit genuine } \\
\text { signature } \\
-93.4 \% \text { fake signatures could have been recognized from } \\
\text { real. and only } 6.6 \% \text { could not have been detected. For } \\
\text { verification, we used } 5 \text {-fold cross-validation, with mean of } \\
\text { EER } 1 / 43.57 \text { and standard deviation of EER } 1 / 40.736 \text {. }\end{array}$ \\
\hline $\begin{array}{ll}\text { Kunal ,kiran } & \text { and } \\
\text { Mrs.sunitajadhav.[10]. }\end{array}$ & 2018 & Eye task & $\begin{array}{l}\text {-Successful computer access for people with disabilities. } \\
\text {-Serving patients with special needs. } \\
\text {-eyetask does not have the contents to be transferred by } \\
\text { the user. }\end{array}$ \\
\hline
\end{tabular}

\section{Discussion:-}

Through the previous comparison, several different conclusions were reached in terms of improvement, performance, accuracy, speed and cost. In paper[4] we find that automatic signature verification systems are very important and give high accuracy similar to the accuracy of the rest of the biometric systems. The verification process was done without relying on sensors. Paper[5] also improved the speed of signature verification by means of eye tracking technology as the system allowed for individual errors such as missing, inserted or alternate characters. Positive results were also obtained in eye tracking technique using the eyenalysis algorithm in the paper[6] also improved the use of eye tracking as a data collection tool. Paper [10] also developed and improved eye tracking techniques by providing an effective service for people with disabilities to interact with computers, Paper [9] also presented good safety and ease of use Eyepass shapes but slightly slower The paper [8] also provided ways to complete signature verification techniques depending on the physiological person's muscles during signing and easily detecting between real and fake signatures, The paper[7] also presented improvements to the quality and performance of signature verification systems using kinetic theory For human movement.

\section{Conclusion:-}

A comparison of this paper found that there are many ways to make a digital signature, some of them are based on biometric features, others are not based on biometric features, and the system based on biometric features has an additional level of protection and is more powerful. Some digital signature systems based on biometric features Which cannot be imitated and difficult to forget such as signature based on biometric authentication, eye movement tracking, eye function, eye movement with biometric features, eye details, eye looks, physiological characteristics of hand and tendon bones, kinetic theory of movements As mentioned in Table 1, it was found that the digital signature on eye tracking is not always a high security but is intended to help people with special needs rely on the movement of their eyes to determine their digital signatures as well as to interact with them easily. 


\section{Reference:-}

1. B.Roman, K. Tomi, M. Andrei and F.Pasi : Eye-Movements as a Biometric, H. Kalviainen: SCIA, LNCS 3540, pp. 780-789, Springer-Verlag Berlin Heidelberg 2005.

2. A.Carlos, L.Francisco and F. Rogelio: Biometric authentication based on eye movements by using scan-path comparison algorithms, Quintana-Nev'arez is a undergraduate student of the Software Engineering programmer at UACJ.2017

3. Z.Youmingand J.Martti: On Biometrics with Eye Movements IEEE Journal of Biomedical and HealthInformatics, vol. 21, no.5, pp.1360-1366 2017.

4. P.Srikanta, P.Umapada and B.Michael:Signature-based Biometric Authentication,School of Information and Communication Technology,Griffith University, Gold Coast, Australia2.CVPRU, Indian Statistical Institute, Kolkata, India S Pal, U Pal, M Blumenstein - Computational Intelligence in Digital..,Springer2014.

5. W. Justin, M. Kenrickand H.Bogdan:Gaze-Based Password Authentication throughAutomatic Clustering of Gaze Points,2011.

6. Bruneau, Daniel, M. Angela Sasse, and J. D. McCarthy. "The eyes never lie: The use of eye tracking data in HCI research." Proceedings of the CHI. Vol. 2. 2002.

7. F. Andreasand P. R'ejean:Signature Verification Based on the KinematicTheory of Rapid Human Movements, This article has been accepted for inclusion in a future issue of this journal. Content is final as presented, with the exception of pagination, 2016.

8. G. Yousef, M.Keivan, N. A Motie and Sh.M.Mohammad: A new method for signature verification based on physiological characteristics of hand muscles and tendons.Biomedical Engineering: Applications, Basis and Communications, Vol. 29, No. 11750006 (8 pages) 2017.

9. D.Alexander, D.Martin and H.Heinrich: Look into my eyes! Can you guess my password ?Symposium on Usable Privacy and Security (SOUPS), CA USA. July 15-17, 2009.

10. A.K.Kunal,B.K.KiranandJ.Sunita: A Novel Approach to Help People Interact with Their SurroundingThrough Their Eyes, IEEE 18th International Conference on Advanced Learning Technologies 2018.

11. H. Shaimaa,A. Eqbasand A.IsraaAhmed :Security Systems Based On Eye Movement Tracking Methods, Journal of AL-Qadisiyah for computer science and mathematics, ISSN (Print): 2074 - 0204 ISSN (Online): 2521 - 3504, Vol.10 No.3 Year 2018. 